\title{
Investigation of Linear Momentum and Impulse Using Video Analysis
}

\author{
L. Kholida, F. D. E. Latief \\ Master of Physics Teaching, Faculty of Mathematics and Natural Sciences \\ Institut Teknologi Bandung \\ Bandung, Indonesia \\ liyakholida92@gmail.com
}

\begin{abstract}
This study aims to present the use of Tracker software as a pedagogical tool for conducting experiments by means of video-based analysis for three different type of collisions using air track apparatus. Video-based analysis has the possibility to provide a better way of visualizing the motion for a direct inspection, especially by using a high-speed camera (some of the modern ones have the capability of producing more than 1000 fps). The first and second collisions were considered to be inelastic and the third collision was set to be completely inelastic. The results revealed that the momentum and energy do not conserve for the three type of collisions. These indicate that there were external force and energy transformation during the collisions. Tracker provides the ability to present the force versus time curve, in which the magnitude of linear impulse can be analyzed
\end{abstract}

Keywords - tracker software; video-based Analysis

\section{INTRODUCTION}

Experiments on mechanics, including linear momentum and impulse involving various kinds of collisions are commonly conducted using air track apparatus. Sensors are usually used to record the position and time to further calculate the velocity before and after collisions. George et al., (2013) utilize the photogate sensor for teaching energy, momentum, and conservation concepts with computer support in an undergraduate physics laboratory [6] Sensors have limited capability especially in detecting a high rate of speed change. Sensors can measure velocity based on a one-point recorded data.

One approach that can better measure and display the phenomenon of momentum and impulse is the video analysis technique. This method is more cost effective as compared to sensor based data acquisition [15]. Video analysis represents a general class of techniques used to extract physical data from video that has recently become a valuable tool in teaching introductory Physics [2]. The physics behavior of the model from video can be compared directly with that of the realworld [3]. One of the free educational video analysis software available is Tracker (Antimirova \& Maliner-Bolotin, 2009). Tracker is video analyzer that can be used for investigating kinematics parameters including the instantaneous acceleration, the instantaneous speed, and the instantaneous position [9].
Tracker is a modeling software that can analyze the motion of objects from videos. Tracker is possible and easy to use simply by overlaying simple dynamical models onto the videos directly. This model will be automatically synchronized and scaled to the video for direct comparison with the real world [11]. This study aimed to present the use of Tracker software as a pedagogical tool for conducting experiments by means of video-based analysis, with the case study of collisions using air track apparatus.

\section{METHOD}

Air track is a set of experiment apparatus produced by Pudak Scientific ${ }^{\mathrm{TM}}$ which consists of air track with a blower and two gliders. The blower supplies air pressure in the air track, thus the air will be blown out from the tiny holes along the track. The track provides an almost frictionless cushion and raise the glider $\pm 0.1 \mathrm{~mm} \mathrm{[10]}$.

This study discussed three specific experiments, each with a distinct type of collision between the two gliders on a frictionless surface (air track). The first experiment is the elastic collision of moving glider colliding into a stationary glider. In the second experiment, each glider had a steel spring bumper attached which will exert a conservative force on the other glider during the collision. For the third experiment, bumpers on the gliders are replaced by Velcro ${ }^{\circledR}$, thus the gliders stuck together after collision (see Fig. 1 for illustration of the collisions). The collisions were investigated using video analysis software Tracker v.4.92. The collisions were recorded at 30 fps using a pocket digital camera. The camera was positioned in front of the air track at same position for all measurement. During the recording, the camera's field of view is adjusted to capture the area of the collisions.

Video was imported into Tracker software and spatial calibration (spatial scaling) was done consequently using the calibration stick feature and clip settings to establish a scale and reference frame for position data. Tracker examined the video frame-by-frame and tracked the objects of interest automatically using auto-tracker feature [2]. By using the auto-tracker feature, the motion of gliders can be recorded step by step which would produce all necessary kinematics parameters in graph for further investigation [9]. 


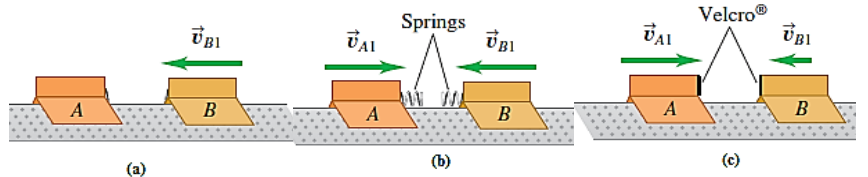

Fig 1. Collisions on a linear air track: (a) stationary glider is collided by moving glider, (b) two moving gliders are collided with a spring, (c) two moving gliders are collided with Velcro® (Sears \& Zemmansky, 2010).

\section{A. Momentum and Impulse}

Figure 1 shows the illustration of two glider collisions with different condition and material. When two bodies collide, momentum is conserved [7]. In contrast, the total kinetic energy of the system may or may not be conserved, depending on the type of collision. In fact, whether or not kinetic energy is conserved is used to classify collisions as either elastic or inelastic [12]. The elasticity of a collision is indicated by the coefficient of restitution $(e)$ in Equation 1 (Enoka, 2008) as follows [4]:

(Speed after collisions $)=-e($ speed before collisions $)$

$$
\begin{aligned}
v_{A}^{\prime}-v_{B}^{\prime} & =-e\left(v_{A}-v_{B}\right) \\
e & =-\frac{v_{A}^{\prime}-v_{B}^{\prime}}{v_{A}-v_{B}} .
\end{aligned}
$$

When two bodies collide, they exert forces with equal magnitude on each other as shown in Equation 1. These forces act in opposite directions:

$$
F_{A}=-F_{B}
$$

If the bodies are in contact for an interval of time $t$, then,

$$
\begin{gathered}
F_{A} t=-F_{B} t \\
I_{\mathrm{A}}=-I_{B} .
\end{gathered}
$$

using the fact that impulse is equal to the change of momentum as shown in Equation 5 (Graham et al., 2002).

$$
\begin{gathered}
I_{\mathrm{A}}=-I_{B} \\
m_{A} v_{A}-m_{A} v_{A}^{\prime}=-\left(m_{B} v_{B}-m_{B} v_{B}^{\prime}\right) \\
m_{A} v_{A}+m_{B} v_{B}=m_{A} v_{A}^{\prime}+m_{B} v_{B}^{\prime} .
\end{gathered}
$$

In this research, Equation 1, 3, 4 and 5 are used to analyze the collision. The experiments are conducted with the assumption that there is no friction on the air track.

\section{RESULTS AND DISCUSSION}

Collisions were analyzed using Tracker. By using Tracker, it is possible to have precise measurements of glider's position at incremental times during its motion and showed it in the graph [1]. The movement can be easily observed by point tracking (blue and red point) as shown in Figure 2 [5]. Figure 3,4 , and 5 were the result of analysis video from tracker. Tracker can show the variety of graph such as force and mo- mentum. The formula can be customized in the tracker with data builder feature.
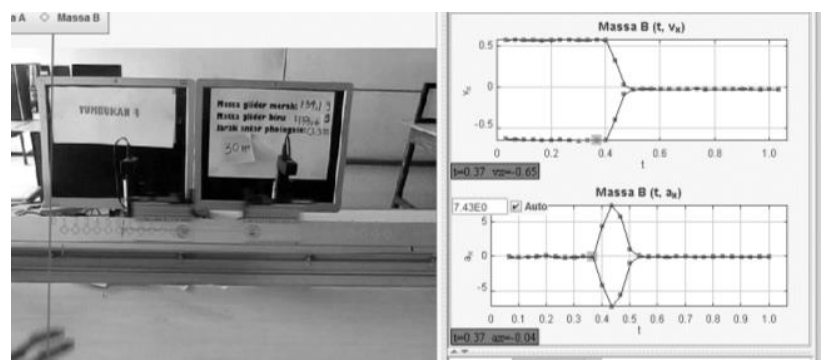

Fig 2. Video analysis of a gliders collision using Tracker

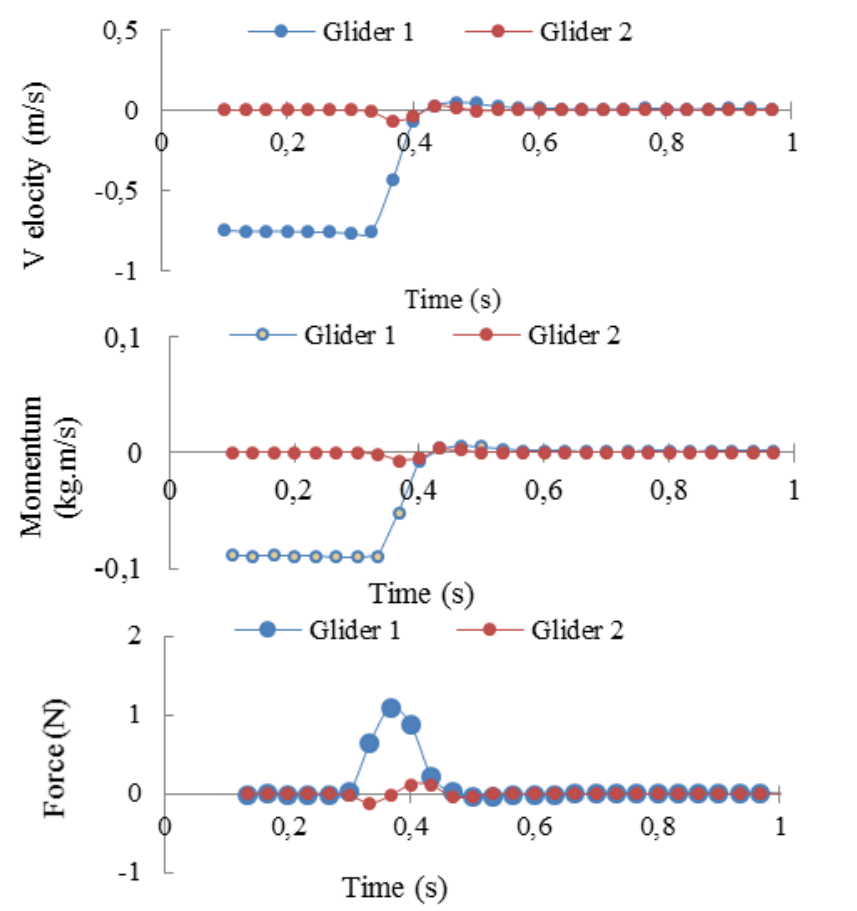

Fig 3. The result of Stationary glider was collided by the moving glider.

The initial velocity of the moving glider is $0.76 \mathrm{~m} / \mathrm{s}$, and then collided with the stationary glider where the stationary glider experienced a little movement (see graph in Fig. 3). However, the stationary glider returned to original state after collision. The final velocity of the moving glider is $0.01 \mathrm{~m} / \mathrm{s}$. The system lose energy after the collision.

Figure 3 shows the moving glider moves slower after collisions, thus the momentum of the system decreased. It indicates that both energy and momentum are not conserved. The system lost $99.97 \%$ of the energy after collision. The magnitude of coefficient of restitution is 0.02 closer to inelastic collision than elastic collision. In an inelastic collision, some of the mechanical energy is dissipated as thermal energy and not all of the kinetic energy is recovered [8]. A collision in which the to- 
tal kinetic energy after the collision is less than before the collision is known as an inelastic collision [13].

TABLE I. THE RESULT OF STATIONARY GLIDER WAS COLLIDED WITH A MOVING GLIDER

\begin{tabular}{|c|c|c|c|}
\hline Glider & $\boldsymbol{v}(\mathbf{m} / \mathbf{s})$ & $\boldsymbol{v}^{\prime}(\mathbf{m} / \mathbf{s})$ & I $(\mathbf{k g . m} / \mathbf{s})$ \\
\hline 1 & -0.76 & 0.01 & 0.09 \\
\hline 2 & 0 & 0 & 0 \\
\hline
\end{tabular}

TABLE II. THE RESULT OF STATIONARY GLIDER WAS COLLIDED WITH A MOVING GLIDER

\begin{tabular}{|c|c|c|c|c|}
\hline $\boldsymbol{P}(\mathbf{k g . m} / \mathbf{s})$ & $\boldsymbol{P}^{\prime}(\mathbf{k g . m} / \mathbf{s})$ & $\boldsymbol{E}(\mathbf{J})$ & $\boldsymbol{E}^{\prime}(\mathbf{J})$ & $\boldsymbol{e}$ \\
\hline & & & & 0.0 \\
-0.09 & 0.002 & 0.03 & 0 & 2 \\
\hline
\end{tabular}

mass of the gliders $=0,12 \mathrm{~kg}$ energy loss: $99.97 \%$

In the second experiment, kinetic energy was stored as potential energy in the compressed springs during the collision. Spring bumper provides a means of generating a conservative force so that no mechanical energy is lost or gained during the collision [13]. But the result was off from what the theory expected to show for an elastic collision: there is energy loss of about $44 \%$. Figure 4 showed initial and final velocity, momentum and force. After collision, the final velocity and momentum of each glider is decreased. The final total momentum in the system is greater than the initial. That indicated, the momentum is not conserved.

TABLE III. THE RESULT OF THE COLLISION OF TWO MOVING GLIDERS WITH SPRING

\begin{tabular}{|c|c|c|c|}
\hline Glider & $\boldsymbol{v}(\mathbf{m} / \mathbf{s})$ & $\boldsymbol{v}^{\prime}(\mathbf{m} / \mathbf{s})$ & I $(\mathbf{k g} . \mathbf{m} / \mathbf{s})$ \\
\hline 1 & -0.43 & 0.29 & 0.09 \\
\hline 2 & 0.39 & -0.24 & -0.08 \\
\hline
\end{tabular}

TABLE IV. THE RESULT OF THE COLLISION OF TWO MOVING GLIDERS WITH SPRING

\begin{tabular}{|c|c|c|c|c|}
\hline$P(\mathrm{~kg} . \mathrm{m} / \mathrm{s})$ & $P^{\prime}(\mathrm{kg} . \mathrm{m} / \mathrm{s})$ & $E(\mathrm{~J})$ & $E^{\prime}(\mathrm{J})$ & $e$ \\
\hline-0.005 & 0.006 & 0.021 & 0.012 & 0.7 \\
\hline
\end{tabular}

mass of gliders $=0.13 \mathrm{~kg}$ energy loss: $44 \%$

For the third experiment, bumpers on the gliders were replaced by $\operatorname{Velcro}{ }^{\circledR}$, so the gliders stuck together after collision. The result as shown in Figure 5 is completely inelastic collision because the gliders stuck together. After collision, the final velocity of each glider was the same: $-0.03 \mathrm{~m} / \mathrm{s}$. The negative direction due to the energy of glider 2 (the direction of negative) was greater than that of the glider 1 .

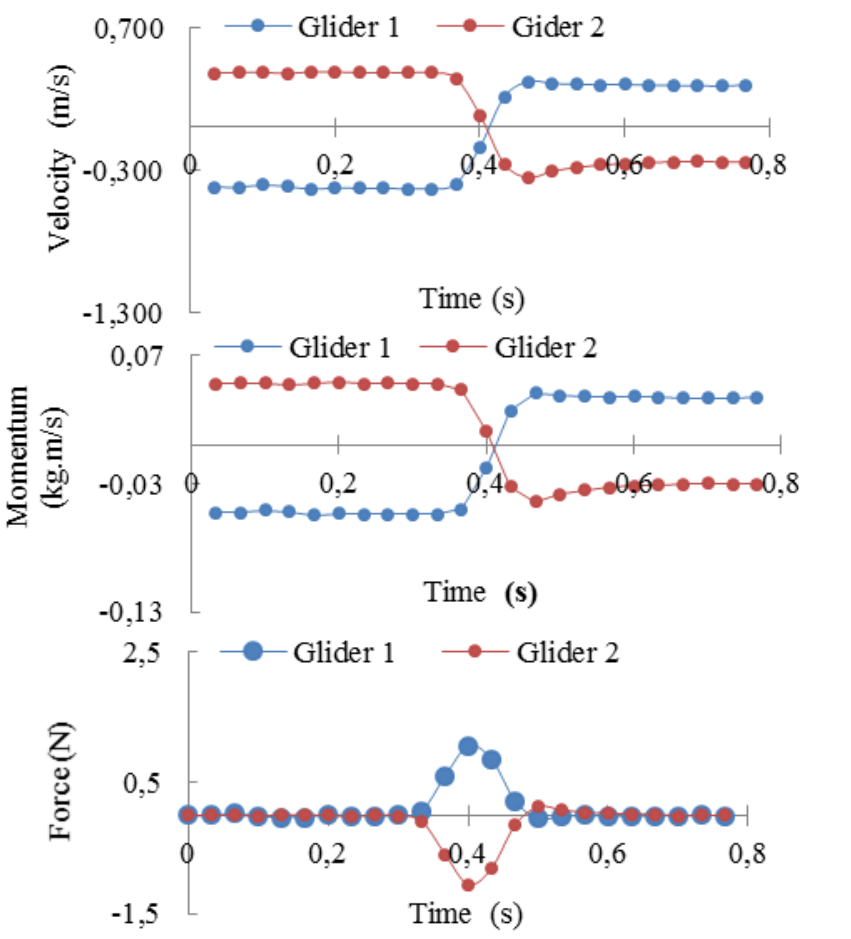

Fig 4. The result of two moving gliders was collided with a spring

The final energy lost from the system is $99.8 \%$ but the final total momentum of the system was greater than its initial. These indicated that there was no conservation of energy nor momentum in this system.

There were no elastic collisions occurred for all the three experiments. It was not easy to set up an ideal system despite using air track with minimal friction. It was shown that the final energy for all the experiments was less than the initial energy, because the kinetic energy is converted into other forms such as heat, sound, friction etc. [14]. That circumstance with the energy was in contrast with the momentum. The final momentum was somehow greater than that of the initial one thus the momentum was not conserved. The conservation energy will be possible if the system is perfectly isolated $\left(F_{\text {net }}=\right.$ $0)$ or within which the interactions are sufficiently short and intense that external forces is ignored for the duration of the interaction (the impulse approximation). Isolated system occurs if the system is not influenced or altered by external forces from the environment [8]. 

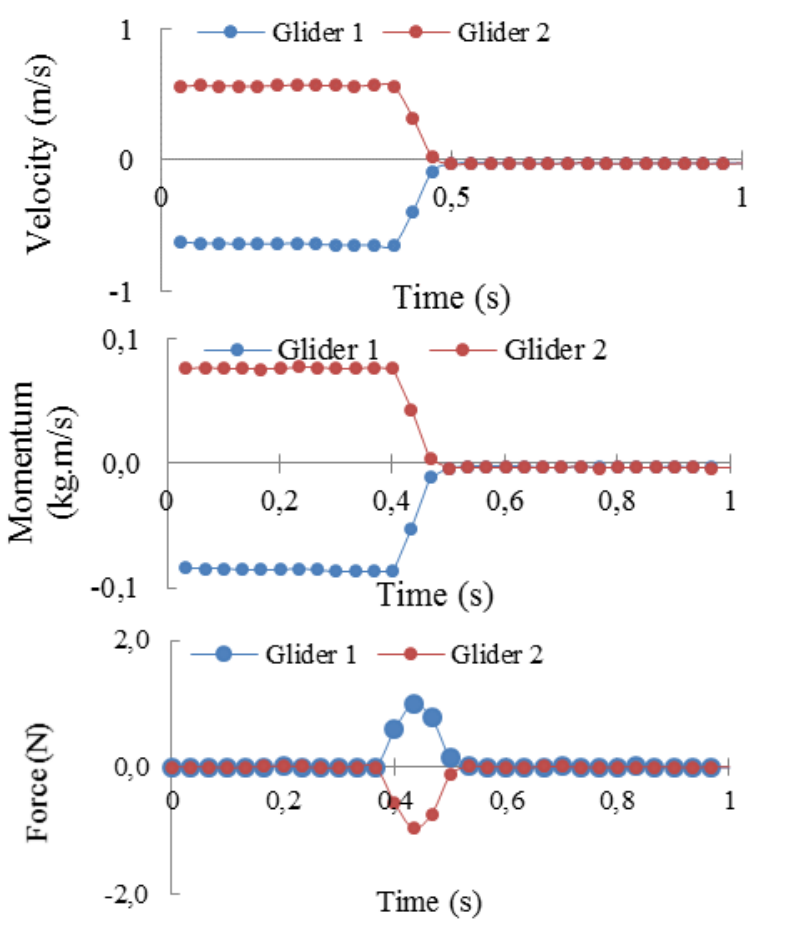

Fig 5. The result of two moving gliders was collided with a Velcro®

If the force acts over time, interval from $t_{\mathrm{i}}$ to $t_{\mathrm{f}}$, that delivers an impulse changes the object's momentum. The geometric interpretation of impulse was shown in Figure 3, 4, and 5 for the area under the $F_{x}$ - versus - $t$ graph. From the graph, if the initial velocity of glider was positive, the Force of glider was negative and if the initial velocity of glider was negative, the force of glider was positive. In the theory, the magnitude impulse of each glider is equal but opposite direction. The result shown as in Table 4 and 3, the magnitude of impulse each glider has opposite direction and has almost the same value

TABLE V. THE RESULT OF TWO MOVING GLIDERS WAS COLLIDED WITH A VELCRO ${ }^{\circledR}$

\begin{tabular}{|c|c|c|c|}
\hline Glider & $\boldsymbol{v}(\mathbf{m} / \mathbf{s})$ & $\boldsymbol{v}^{\prime}(\mathbf{m} / \mathbf{s})$ & I $(\mathbf{k g . m} / \mathbf{s})$ \\
\hline 1 & -0.64 & -0.03 & 0.082 \\
\hline 2 & 0.57 & -0.03 & -0.08 \\
\hline
\end{tabular}

TABLE VI. THE RESULT OF TWO MOVING GLIDERS WAS COLLIDED WITH A VELCRO®

\begin{tabular}{|c|c|c|c|c|}
\hline $\boldsymbol{P}(\mathbf{k g . m} / \mathbf{s})$ & $\boldsymbol{P}^{\prime}(\mathbf{k g . m} / \mathbf{s})$ & $\boldsymbol{E}(\mathbf{J})$ & $\boldsymbol{E}^{\prime}(\mathbf{J})$ & $\boldsymbol{e}$ \\
\hline-0.010 & -0.007 & 0.05 & 0 & 0 \\
\hline
\end{tabular}

mass of gliders $=0.13 \mathrm{~kg}$ energy loss $99.8 \%$

\section{CONCLUSION}

Video analysis using Tracker has the capability to show the phenomenon of momentum and impulse in the real time movement and described it in the form of graphical represen- tation simultaneously. The result shown that the momentum and energy did not conserve. These indicate that there were external force and transformation energy after collisions. The first and second collisions were inelastic and the third collision was completely inelastic.

\section{REFERENCES}

[1] J.A. Bryan, Investigating the conservation of me-chanical energy using video analysis: four cases. Physics Education, vol. 45, no. 1, pp. 50, 2010 .

[2] D. Brown and A.J. Cox, "Innovative uses of video analysis," The Physics Teacher, vol. 47, no. 3, pp. 145-150, 2009.

[3] D. Brown, Video modeling: combining dynamic model simulations with traditional video analysis. In American Association of Physics Teachers (AAPT) Summer Meeting, 2008.

[4] R.M. Enoka, "Movement Force", Neuromechanics of Human Movement, 4th ed, U.S: Sheriden Books, ch 2, pp. 72-73, 2008.

[5] P. Hockicko, L.U. Krišt' ák, and M. Němec, "Development of students' conceptual thinking by means of video analysis and interactive simulations at technical universities," European Journal of Engineering Education, vol. 40, no. 2, pp. 145-166, 2015.

[6] E.A. George, M.J. Broadstock and J.V. Abad, "April. Learning energy, momentum, and conservation concepts with computer support in an undergraduate physics laboratory," In Fourth international conference of the learning sciences, pp. 2-3, 2013.

[7] T. Graham, A. Burrows, and B. Gaulter, "Momentum and Collisions in One Dimension", Mechanics 4: Advancing Math for AQA. 1st. USA: Heinemann Educational Publisher, ch 5, pp.71-78.

[8] D.R. Knight, "Impulse And Momentum", Physics For Scientists And Engineers With Modern Physics: A Strategic Approach, AddisonWesley, pp. 261-292, 2017.

[9] E.C. Prima, M. Mawaddah, N. Winarno, and W. Sriwulan, Kinematics investigations of cylinders rolling down a ramp using tracker. In AIP Conference Proceedings (Vol. 1708, No. 1, p. 070010). AIP Publishing, February, 2016.

[10] Pudak Scientifict $\square$. Sistem Percobaan Fisika http://www.pudakscientific.com/image/sistem_percobaan_fisika1(1).pdf, 2017.

[11] M.H. Ramli, K.T. Chan, and W.F. Yapa. Tudy of Simple Pendulum Using Tracker Video Analysis and High Speed Camera: An Interactive Approach to Analyze Oscillatory Motion. Solid State Science and Technology, vol. 224, no. 2, pp. 297 - 305, 2016.

[12] Serway and Jewwet, "Linear Momentum and Collisions", Physics for Scientist and Engineers, 6th ed, U.S: Thomson Brooks, ch 8, 251-292, 2004.

[13] Sears \& Zemansky, "Momentum,Impulse \& Collisions", University Physics in Modern Physics, 13th ed. U.S: Addison-Wesley, ch 8, 241287, 2010.

[14] Stony Brook University, 2017, Brook Physics Laboratory Manuals, http://phylabs1.physics.sunysb.edu/introlabs/labmanual_archive/PHY13 3_Conservation\%20of\%20Momentum.pdf, 2017.

[15] O.S.K.S. Sastri, International Journal of Advanced Information Science and Technology, vol. 27, no. 27, pp. 19-23, 2014. 